Path. Microbiol. 1964;27:I-X

\title{
Contents, Vol. 27, 1964
}
H. CHIARI, Wien
H. COTTIER, Bern
H. FEY, Bern
J. FIRKET, Liège
C. HALLAUER, Bern
E. LETTERER, Tubingen
J. L. NICOD, Lausanne
COLLABORATORES

A. L. OLITZKI, Jerusalem R. H. REGAMEY, Geneve F. C. ROULET, Basel E.

RUTISHAUSER, Geneve M. et R. SILBERBERG, Saint Louis, Mo.

H. SMETANA, Washington J. TOMCSIK, Basel R. TULASNE, Strasbourg E. UEHLINGER, Zurich M. WELSCH, Liège A.WERTHEMANN, Basel

REDACTORES A. GRUMBACH, Zurich J. R. RÜTTNER, Zurich

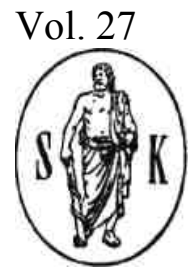

1964

\section{BASEL (Schweiz) S. KARGER NEW YORK}

Alle Rechte, insbesondere das de $\Gamma \mathrm{t}$ ) bersetzung in fremde Sprachen, vorbehalten.

Ohne ausdrückliche Genehmigung des Veriages ist es auch nicht gestattet, dieses Buch oder Teile daraus auf photomechanischem Wege (Photokopie, Mikrokopie) zu vervielfãltigen.

(C)

Copyright 1964 by S. Karger AG., Basel Printed in Switzerland by Buchdruckerei Friedrich

Reinhardt AG., Basel Cliches: Aberegg-Steiner \& Cie. AG., Bern, und Steiner \& Cíe. AG., Basel Lorez, H. P.: Vide Studeb, A.

Lüdin, M.: Myokardveränderungen beim plötzlichen Tod aus natürlicher Ursache 475

Lüscher, E. F.: Vide Bettex-Galland, M.

Magnússon, S.: Leukemia in Iceland 1957 to 1961705

Maier, C.: $\quad$ Vide Kuenzle, C.

Mainwaring, A. R.:

Vide Edington, G. M.

Marek, J.: Vide Koí > ousek, R.

Matkovics, B.; Kovács, E.; Kókay, K.; Fiszter, J. and Zador, S.:

Some Data Concerning Biochemical Properties and Resistance to

Antibiotics in Staphylococci. Preliminary Communication 339

Matkovics, B.: Vide Zador, S. Meier-Ruge, W. und Ebert, L. P.: 
Die Beziehungen der verschiedenen Lipoidfraktionen der menschlichen

Leber zur Verfettungsform derselben 135

Meister, H.: Zur Histopathologie der Niere bei akuter hämorrhagischer und chronisch rezidivierender Pankreatitis 276

Méry, J. Ph. : Vide Berger, J.

Méwissen, D. J. and Betz, E. H.:

Morbidity and Survival Rates for Cancers of the Hematopoietic

System in Belgium 739

Missmahl, H. P. and Gafni, J.:

Peri-Collagen and Peri-Reticular Amyloidoses. Their Differentiation

by Polarization Microscopy 826

Miyamoto, K.: Vide Wakisaka, G.

Morgan, C.: Vide Isliker, $\mathrm{H}$.

Moriga, M.: Vide Wakisaka, G.

Mühlemann, H.: Vide Roggen, G.

Muir, C. S.: Geographical Pathology of Leukaemia in Singapore 760

Muir, C. S. and Thomas, M. A.:

Geographical Pathology of Amyloidosis in Singapore $\quad 848$

Nakagawa, S. and Suzue, K.:

Amyloidosis in Japan 850

Nakamura, T.: Vide Wakisaka, G.

Neuhold, R.: Vide Breitfellner, G.

Nicod, J.-L. et Burgisser, H.:

Lymphomes malins chez le lièvre et le chat

I

Nitschmann, H. und Gygax, H. R.:

Eine einfache Methode zur Messung der relativen onkotischen Wirk-

samkeit von kolloidalen Plasmaersatzlösungen $\quad 548$

Obrecht, P.: Vide Harwerth, H.-G.

Olitzki, A. L.; Godinger, D. and Gershon, Z.:

The Uptake of Salmonella typhi by Phagocytes and its Intracellular

Viability 175

Olsson, R.: $\quad$ Vide Bengmark, S.

Paillas, J.: Vide Delarue, J.

Parnas, J.; Lazuga, K. and Kadziolka, A.:

Experimental Investigations on the Live-Antibrucellosis-Vaccine . 1012 Parnas, J. et Sarnecka, B.:

Plaques de Brucellaphages sur cultures de Brucella suís 334

Index

Agnihothi, V. P.: Studies on Aspergilli. IX. Utilization of Raffinose and its Component

Sugars. A Chromatographic Study 88

Ajello, L. et de Marco, S.:

Sur la distribution, frequence et formes des leucémies observées à $\Gamma \operatorname{lnstitut~d'Anatomie~}$

Pathologique de ГUniversité de Rome .... 753

Ammann, P.: Vide Hässig, A.

Ang, Ö.: $\quad$ Vide Çetin, E. T.

Angeletti, C. A.: Vide de Matteis, A. 
Arendt, A. und Böhm, W.:

Beitrag zur Coxsackie B 4-Enzephalitis $\quad 31$

Avtsyn, A. P.: The Interrelation Between Medical Geography and Geographical

Pathology 943

Babudieri, B. and Farina, R.:

The Leptospirae of the Italian Hedge-Hog 103

Barandun, S.: Vide Diggelmann, H.

Bari, W. A. and Sorenson, G. D.:

Ultrastructural Alterations in X-Irradiated Spleen 257

Battaglia, S.: Results of a Statistical Investigation on Amyloidosis 792

Becla, E.: Development of Paper Chromatograms of Growth Factors by a Bio

logical Method 232

Bengmark, S. and Olsson, R.:

The Effect of Castration and Testosterone Treatment on Liver

Healing in Male Rats after Carbon Tetrachloride Injury 167

Berchtold, H.: Vide Roggen, G.

Berger, J.; Hinglais, N. et Méry, J. Ph.:

L'amyloïdose rénale. Experience d'un service de néphrologie parisien 878

Bernard, J.: Etude générale de la géographie des leucémies 708

Bettex-Galland, M. und Lüscher, E. F.:

Untersuchungen über die Auslösung der viskösen Metamorphose der

menschlichen Blutplättchen durch Immunkomplexe 533

Betz, E. H.: Vide Méwissen, D. J.

Böhm, W.: Vide Arendt, A.

Bonifas, V. H.: Vide Zwillenberg, L. 0.

Borel, J. F.: Vide Hässig, A.

Breitfellner, G. und Neuhold, R.:

Therapieeffekte und Komplikationen bei cytostatischer und Cortison-

therapie bei Leukosen 777

Breitfellner, G. und Neuhold, R.:

Amyloidose bei tuberkulösem Primärkomplex und Miliartuberkulose 863

Brochériou, $\mathrm{Cl}$.: Vide Chomette, G.

Brzin, B.: $\quad$ Slimy Growth of Bacterial Colonies Exposed to Antibiotics .... 347

Bbzin, B.: Microbial Antagonism by Pseudomonas aeruginosa on Pasteurella pseudotuberculosis 353

Bbzin, B.: Dependency of Cell Size and Form of Proteus mírabílis on the Peptone

in the Culture Medium 1021

Brzin, B.: Synergistic Action of the Unfavourable Incubation Temperature and

Sulphathiazol on the Cells of Bacterium anítratum 1035

Burgisser, H.: Vide Nicod, J.-L.

Butler, B. und Greuter, W.:

Zwei weitere Fälle der Mutter/Kind-Kombination Gm $(\mathrm{a}-\mathrm{b}+\mathrm{)})$

$\mathrm{Gm}(\mathrm{a}+\mathrm{b}-) \quad 558$

Butler, B.: Vide Hässig, A.

Çetín, E. T.; AnG, Ö.; Kasimoglu, Ö.; Ersoy, M. and Gence, H.:

State of Susceptibility to Antibiotics of 832 Bacterial Strains Isolated 
Chiappino, G. and Pernis, B.:

Demonstration with Immunofluorescence of 19S Macroglobulins and 7 S Gamma Globulins in Different Cells of the Human Spleen. (With plate I.) 8

Chomette, G.; Pinaudeau, Y.; Ganter, P. et Brochériou, Cl. :

Note à propos de $\Gamma$ amyloïdose cardiaque du vieillard 872

Chomette, G.: Vide Delarue, J.

Costa, A. and Weber, G.:

Cirrhosis, Adenocirrhosis, Cancrocirrhosis 947

Costin, I. D.; Voiculescu, D. and Gorcea, V.:

An Outbreak of Food Poisoning in Adults Associated with Escherichía colí serotype 86: B7: H34 68

Cottier, H.; Keller, H. und Roos, B.:

Generalisierte Hyperostosis interna und Osteosarkome bei röntgenganzbestrahlten weiblichen Schweizer Albinomäusen mit hormonal aktiven Ovarialtumoren 458

Court Brown, W. M.; Doll, R. and Hill, I. D.:

Leukaemia in Britain and Scandinavia 644

Da Silva Horta, J.; Filipe, I. and Duarte, S.:

Portuguese Polyneuritic Familial Type of Amyloidosis $\quad 809$

Davies, A. M.: Vide Rosenmann, E.

Delarue, J.; Paillas, J.; Chomette, G. et Ganter, P.:

Les tumeurs amyloïdes à localisation trachéo-bronchique exclusive 867

De Marco, S.: Vide Ajello, L.

De Matteis, A. and Angeletti, C. A.:

Primary Fibrosarcoma of the Lung 129

Dietrich, F. M.: Antikörperbildung gegen löslich, aggregierte, präzipierte und adsorbierte Proteine in C57BL/6-Mäusen 356

Dietrich, F. M.: The Immune Response of C57BL/6 Mice to Protein Antigens Incor porated into Freund's Incomplete Adjuvant 1025

Diggelmann, H.; Koblet, H.; Gerbeb, H. und Babandun, S.:

Bestimmung der totalen Clearance von Standard-Gammaglobulin, pH-4-Gammaglobulin und Gamma-Venin 572

Doll, R.: $\quad$ Vide Court Brown, W. M.

Dreifuss, J. J.: Vide Girardier, L.

Duarte, S.: $\quad$ Vide da Silva Horta, J.

Ebert, P. L.: Vide Meier-Ruge, W.

Edington, G. M. and Mainwaring, A. R.:

Amyloidosis in Western Nigeria 841

Eridani, S. and Tiso, R.:

Incidence and Distribution of the Cases of Leukemia in the Years

1959-1961 in the Province of Milan 746

Ersoy, M.: Vide Çetin, E. T.

Farina, R.: Vide Babudieri, B.

Fernandez-Criado, M.: 
Some Observations on Renal Amyloidosis. A Clinical, Histopathologic

and Biochemical Experience 880

Filipe, I.: Vide da Silva Horta, J.

Fischer, F.: $\quad$ Die Untersuchung des Wohnsitzes leukämischer Kinder 767

Fiszter, J.: Vide Matkovics, B.

Fossati, C.: L'amyloïdose dans la tuberculose pulmonaire chez les Arabo-Lybiens

de la Cyrénaïque avant et après $\Gamma$ emploi des chimio-antibiotiques. . 856

Foukas, M.: Vide Töndury, G.

Frey-Wettstein, M.:

Befall der Bantu-Bevölkerung Ost-TransvaaГs mit malignen Tumoren 494

Gafni, J.: Vide Heller, H.

Gafni, J.: Vide Missmahl, H. P.

Ganter, P.: Vide Chomette, G.

Ganter, P.: Vide Delarue, J.

Gazenfield, E.: Vide Rosenmann, E.

Gence, H.: Vide Çetin, E. T.

Gerber, H.: Vide Diggelmann, H.

Gershon, Z.: Vide Olitzki, A. L.

Girardier, L.; Dreifuss, J. J.; Haenni, B. et Petrovici, A.:

Réponse du tissu myocardique de rat in vitro à une augmentation de

la pression osmotique du milieu externe

16

Godinger, D.: Vide Olitzki, A. L.

Gorcea, V.: Vide Costin, I. D.

Von Graevenitz, A.:

Über Serratia-Infektionen beim Menschen 235

Greuter, W.: Vide Butler, R.

Gunz, F. W.: Leukaemia in New Zealand and Australia 697

Gygax, H. R.: Vide Nitschmann, H.

Haenni, B.: Les effets de Гintoxication aiguë au formiate d'allyl sur le foie de rat.

Etude au microscope électronique 974

Haenni, B.: Vide Girardier, L.

Harwerth, H.-G.; Reissner, I.; Obrecht, P.; Trier, H. G. und Heilmeyer, L.:

Zur geographischen Pathologie der Leukämien: Ergebnisse der Um-

frage in Zentraleuropa 655

Hässig, A.; Borel, J. F.; Ammann, P.; Thöni, M. und Butler, R.:

Essentielle Hypokomplementämie 542

Heilmeyer, L.: Vide Harwerth, H.-G.

Heller, H.; Sohah, E. and Gafni, J.:

Classiñcation of Amyloidosis with Special Regard to the Genetic Types 833

Hermanek, P. J.: Quantitative Morphology in Inflammatory Myocardial Changes in

Experimental Animals $\quad 157$

Hill, I. D.: Vide Court Brown, W. M.

Hinglais, N.: Vide Berger, J.

Illés, J.: $\quad$ Die bestgeeigneten Plasmen für die Staphylokoagulasereaktion . 117

Illés, J.: $\quad$ Zum Nachweis der Antikoagulase bei Staphylokokkeninfektionen. . 343

Isliker, H.; le Maire, B. and Morgan, C.: 
The Use of Ferritin-Conjugated Antibody-Fragments in Electron-

Microscopic Studies of Viruses

521

Jáki, Á.: $\quad$ Vide KovÁcs, E.

Kadziolka, A.: Vide Parnas, J.

Kallner, G.: $\quad$ Patterns of Leukaemia in Israel 666

Kasimoglu, Ö.: Vide Çetin, E. T.

Keller, H.: Vide Cottier, $\mathrm{H}$.

Kistler, P. und Zahler, P.:

Probleme bei der Herstellung intravenös verabreichbarer, humaner

Gamma-Globulin-Lösungen 564

Koblet, H.: Vide Diggelmann, H.

Kodousek, R.; Marek, J. and Kodousková, V.:

Observations concerning Pancreatic Insular and Renal Tubular Calcifi

cation in Experimental Rats treated with some Calcifying Factors

and $\mathrm{A}$ Пoxan 957

Kodousková, V.: Vide Kodousek, R.

Kókay, K.: Vide Matkovics, B.

Kontbohb, T.: Vide Rauss, K.

KovÁcs, E. und Jáki, Á.:

Eine Methode zur elektrometrischen Bestimmung der Dehydrogenase-

Aktivität $\quad 373$

KovÁcs, E.: Vide Matkovics, B.

KovÁcs, E.: Vide Zadob, S.

Kbuml, J.: Vide Urbancík, R.

Kuenzle, C; Maieb, C. und Rüttneb, J. R.:

Ergebnisse einer verbesserten Bilirubin-Chromatographie bei ver-

schiedenen klinischen Ikterusformen 410

Kuenzle, C.: Vide Rüttneb, J. R.

Kuipebs, F. C.: Eosinophilic Phlegmonous Inflammation of the Alimentary Canal

Caused by a Parasite from the Herring 925

Lalich, J. J.: Influence of Rat Strain on Pulmonary Vascular Responses to Monocrotaline Feeding $\quad 965$

Laufeb, A.: Vide Rosenmann, E.

Lazuga, K.: Vide Pabnas, J.

Lee, J. A. H.: Seasonal Variations in the Incidence of the Clinical Onset of Leu

kaemia $\quad 772$

Lehneb, T.: Changing Trends in Amyloidosis since the Introduction of Modern

Chemotherapy 855

Lettebeb, E.: Zur Problematik der Amyloidose 782

Le Maibe, B.: Vide Isliker, $H$.

Loosli, R.: $\quad$ Erzeugung fötaler Mißbildungen am Kaninchen mit Thalidomid . 1003

Pernis, B.: Vide Chiappino, G.

Petrovici, A.: Vide Girardier, L.

Pijper, A. $\dagger$ and Steynberg, A.:

Flagellation of Azotobacters and other Bacteria

1037

Pinaudeau, Y.: Vide Chomette, G. 
Polenská, H.: Vide Urbancík, R.

Rauss, K. and Kontrohr, T.:

Antigenic Structure of Sh. sonneí 310

Reber, K.: $\quad$ Vide Studer, A.

Reissner, I.: Vide Harwerth, H.-G.

Rickenbacher, J.: Infektionsversuche mit Vakzinevirus an menschlicher embryonaler

Haut in vitro 624

Roggen, G.; Berchtold, H. und Mühlemann, H.:

Nachweis der biologischen Wirksamkeit von aus Kunststoífen ex-

trahierten Substanzen an Elritzen 593

Rondez, R.: Zur Vermehrung von Kernvolumen und DNS-Gehalt bei chronischer, toxisch bedingter Leberzellschädigung $\quad 429$

Roos, B.: $\quad$ Vide Cottier, $\mathrm{H}$.

Rosenmann, E.; Gazenfield, E.; Laufer, A. and Da vies, A. M.:

Isoproterenol Induced Myocardial Lesions in the Immunized and Non-Immunized Rat. 1.

Histopathological Lesions in Normal Rats . . 303

Rüttner, J. R.: Die pathologische Anatomie der Leukosen. Auswertung der patho-logischanatomischen Enquête 1957-1961 und Beitrag zur Stellung der Leukosen unter den neoplastischen Proliferationen des RES . 723

Rüttner, J. R.; Spycher, M. A. und Kuenzle, C.:

Zur Pathologie des Ikterus. Der ANIT-induzierte Ikterus der Ratte, ein Modell einer durch Zellmembranschädigung bedingten toxischen

Hepatose 403

Rüttner, J. R.: Vide Kuenzle, C.

Rüttner, J. R.: Vide Spycher, M. A.

Sakurai, M.: Vide Wakisaka, G.

Sarbhoy, A. K.: Nutritional Studies on Six Members of the Mucorales-II. I. Utili

zation of Oligo- and Polysaccharides 216

Sarnecka, B.: Vide Parnas, J.

Schmidtmann, M.: Zur Frage der Entstehung des Bronchialkrebses. Haben wir im

Zigarettenrauchen die Ursache entdeckt? 935

Schnack, H.: Zur Aetiologie des Chlorpromazin-Ikterus: Tierexperimentelle und

klinische Beobachtungen 419

Schnyder, U. W.: Zur Histogenetik der granulösen Degeneration 486

Seeliger, H. P. R.: Vide Werner, H.

Seno, S.: $\quad$ Statistical Investigation of Leukaemia Autopsies in Japan 684

Sforza, M.: $\quad$ On the Distribution of Human Tumours in Eritrea 952

Silberberg, R. and Silberberg, M.:

Pathogenesis of Osteoarthrosis 447

Sohah, E.: Vide Heller, H.

Sorenson, G. D.: Vide Bari, W. A.

Spycher, M. A. und Rüttner, J. R.:

Zur Pathologie des Ikterus. Elektronenmikroskopische Untersuchun-gen über die

Frühveränderungen der Leberparenchymzellen beim alpha-naphtyl-isothiocyanat induzierten

Ikterus der Ratte .... 387

Spycher, M. A.: Vide Rüttner, J. R. 
Steiner, P. E.: Evolution of Research in the Etiological Types of Cirrhotic Diseases of the Liver, 1931-1961 890

Steynberg, A.: Vide Pijper, A.

Stofer, A. R.: Die Früherfassung des experimentellen Herzinfarktes der Ratte mit der TTC-Reaktion 467

Studer, A.; Rebeb, K. und Lobez, H. P.:

Experimentelle Untersuchungen zur Frage der Bedeutung intravasaler

Fibrinabscheidung für die Entstehung arteriosklerotischer Wandver-

änderungen 287

Suzue, K.: $\quad$ Vide Nakagawa, S.

Symeonidis, A.: Primary Cancer of the Liver in Greece 931

Tiso, R.: $\quad$ Vide Eridani, S.

Thomas, M. A.: Vide Muir, C. S.

Thöni, M.: Vide Hässig, A.

Töndury, G. und Foukas, M.:

Die Gefährdung des menschlichen Keimlings durch Pockenimpfung

in graviditate 602

Trier, H. G.: Vide Harwerth, H.-G.

Trnka, L.: Vide Urbancík, R.

Uchino, H.: Vide Wakisaka, G.

Urbancík, R.; Trnka, L.; Kruml, J. und Polenská, H.:

Antimycobakterielle Aktivität von Isoxyl. II. Versuche an Meer-

schweinchen und Kaninchen 79

Vogel, A.: $\quad$ Feinstrukturelle Charakteristika von Kollagenfasern 436

Voiculescu, D.: Vide Costin, I. D.

Vogt, H.: $\quad$ Untersuchungen über die Züchtung von Osteoklasten in der Gewebe-

kul·tur und in Diffusionskammern $\quad 42$

Von Wattenwyl, N.:

Neuere Differenzierungsmethoden der Langerhans'schen Inseln. Ihre Bedeutung für die morphologische Diagnose des Diabetes mellitus . 144

Wakisaka, G.; Uchino, H.; Yasunaga, K.; Nakamura, T.; Sakurai, M.; Miyamoto, K.;

Yoshino, T. and Moriga, M.:

Statistical Investigations of Leukaemia in Japan from 1956 to 1961671

Waser, P. G.: Antagonismus von Curarin/Acetylcholin and Curarin/Kalium in der

Endplatte $\quad 499$

Weber, G.: $\quad$ Vide Costa, A.

Werner, H. und Seeliger, H. P. R.:

Vergleichende Untersuchungen an Bifidus-Stämmen verschiedener

Herkunft 202

WINTERHALTER, K. H.:

Hemoglobin Synthesis 508

Wood, E. E.: Survey of Leukaemia in the West Cornwall Clinical Area (1948-1961) 736

Yasunaga, K.: Vide Wakisaka, G.

Yoshino, T.: Vide Wakisaka, G.

Zador, S.; Matkovics, B. and KovÁcs, E.: 
Variations in Redox Potential Values Caused by Differently Shaped

Electrodes 225

Zador, S.: Vide Matkovics, B.

Zahler, P.: $\quad$ Vide Kistler, P.

Zwillenberg, L. 0. and Bonifas, V. H.:

The Ultrastructure of Stegasma

95

Gesellschaftsberichte - Society Transactions - Sociétés

Proceedings of the Eight Conference of the International Society of Geographical Pathology,

Milano, September 19th-21st, 1963 . . .641

Personalia Prof. A. von Albertini zum 70. Geburtstag 385

Buchbesprechungen - Book Reviews - Livres nouveaux .... 124, 248, 381, 638, 956

Varia 256, 640, 956

Index rerum 1051

Index autorum 1069 